\title{
Conhecimento dos profissionais de enfermagem na avaliação da dor neonatal em uma unidade de terapia intensiva
}

\author{
Knowledge of nursing professionals in the evaluation of neonatal pain in an intensive care \\ unit
}
Conocimiento de los profesionales de enfermería en la evaluación del dolor neonatal en una unidad de terapia intensiva

Larissa Santana de Souza Pereira ${ }^{1 \star}$, Thais de Paula Lima Mendes ${ }^{2}$, Rebeca Pereira da Rocha ${ }^{3}$.

\section{RESUMO}

Objetivos: Avaliar o conhecimento dos profissionais de enfermagem sobre o manejo e correta avaliação da dor em uma unidade neonatal, com o intuito de tornar a assistência mais humanizada e sensibilizar a equipe quanto a importância da dor. Métodos: Pesquisa descritiva qualitativa. Inicialmente foi aplicado um questionário sobre dor. As respostas do questionário foram estabelecidas em 5 categorias por análise de conteúdo. Posteriormente foi realizado uma capacitação através de rodas de conversa fim de tornar a equipe de enfermagem apta em avaliar a dor corretamente. Resultados: A dor continua sendo um tema ainda pouco discutido na prática clínica, além do baixo conhecimento por parte da maioria dos profissionais sobre parâmetros de avaliação da dor específicas em neonatal. Entretanto, na prática, as profissionais mostraram conhecimento em identificar a dor e intervir com medidas de prevenção para o seu alívio. Conclusão: $A$ aplicação do questionário permitiu avaliar o conhecimento da equipe sobre o assunto e assim trabalhar conforme as reais necessidades demonstradas. Através da capacitação também foi possível treinar a equipe para identificar a dor corretamente e, consequentemente, realizar medidas para o seu alívio, tornando assim a assistência em saúde mais qualificada e humana.

Palavras-chave: Capacitação de equipe, Conhecimento, Dor, Enfermagem neonatal.

\begin{abstract}
Objectives: To evaluate nursing professionals' knowledge about the management and correct evaluation of pain in a neonatal unit, in order to make the care more humanized and to make the team aware of the importance of pain. Methods: Qualitative descriptive research. Initially a questionnaire on pain was applied. The responses of the questionnaire were established in 5 categories by content analysis. Subsequently a training was conducted through conversation wheels to make the nursing team apt to evaluate the pain correctly. Results: Pain continues to be a subject that is still little discussed in clinical practice, in addition to the low knowledge by the majority of professionals about specific neonatal pain assessment parameters. However, in practice, the professionals showed knowledge in identifying the pain and intervening with prevention measures for their relief. Conclusion: The application of the questionnaire allowed to evaluate the knowledge of the team on the subject and thus to work according to the real needs demonstrated. Through training, it was also possible to train the team to identify pain correctly and, consequently, to take measures for their relief, thus making health care more qualified and humane.
\end{abstract}

Key words: Staff training, Knowledge, Pain, neonatal nursing.

\footnotetext{
${ }^{1}$ Escola Superior de Ciências da Saúde do Distrito Federal. *E-mail: larisantss@gmail.com

${ }^{2}$ Grupo Atualiza especializado em cursos e treinamentos abertos e in company nas áreas de Saúde.

${ }_{3}$ Universidade Estadual de Londrina -UEL e Universidade de Brasília -UnB.
} 


\section{RESUMEN}

Objetivos: Evaluar el conocimiento de los profesionales de enfermería sobre el manejo y correcta evaluación del dolor en una unidad neonatal, con el objetivo de hacer la asistencia más humanizada y sensibilizar al equipo en cuanto a la importancia del dolor. Métodos: Investigación descriptiva cualitativa. Inicialmente se aplicó un cuestionario sobre el dolor. Las respuestas del cuestionario se establecieron en 5 categorías por análisis de contenido. Posteriormente se realizó una capacitación a través de ruedas de conversación para hacer que el equipo de enfermería apto en evaluar el dolor correctamente. Resultados: El dolor sigue siendo un tema aún poco discutido en la práctica clínica, además del bajo conocimiento por parte de la mayoría de los profesionales sobre parámetros de evaluación del dolor específicos en neonatal. Sin embargo, en la práctica, las profesionales mostraron conocimiento en identificar el dolor e intervenir con medidas de prevención para su alivio. Conclusión: La aplicación del cuestionario permitió evaluar el conocimiento del equipo sobre el asunto y así trabajar conforme a las reales necesidades demostradas. A través de la capacitación también fue posible entrenar al equipo para identificar el dolor correctamente y, consecuentemente, realizar medidas para su alivio, haciendo así la asistencia en salud más calificada y humana.

Palabras clave: Capacitación de equipo, Conocimiento, Dolor, Enfermería neonatal.

\section{INTRODUÇÃO}

O nascimento de um Recém-Nascido Pré-Termo (RNPT) requer a disponibilidade de um suporte clínico especializado que seja capaz de suprir todas as fragilidades dessa faixa populacional frente a sua instabilidade clínica. Nesse contexto, a Unidade de Terapia Intensiva Neonatal (UTIN) caracteriza-se como o ambiente hospitalar mais adequado para esses recém-nascidos $(\mathrm{RN})$ por ter disponível todos os recursos tecnológicos, humanos e terapêuticos dos quais necessitam. Tal fato tem reflexos positivos como o aumento na sobrevida orgânica dos prematuros (SANTOS LM et al., 2012).

Entretanto, a hospitalização do RNPT na UTIN, por estar associada a um número excessivo de procedimentos clínicos, pode gerar no RN desconforto, estresse e dor. Frente a isso, faz-se importante a utilização de medidas que contribuam à amenização desses fatores visando uma recuperação satisfatória de seu estado de saúde· (SANTOS LM et al., 2012).

A dor deve ser considerada como o quinto sinal vital; sendo assim deve ser avaliada como tal e realizadas as devidas intervenções quando necessário para o seu controle. No RN torna-se mais difícil a mensuração desse parâmetro frente à impossibilidade de comunicação verbal dessa população. Nesse contexto, a utilização de instrumentos ou indicadores que auxiliem tanto a qualificação como a quantificação da dor contribui para a melhoria no manuseio da dor nesses pacientes (TAMEZ RN, 2013).

A dor é uma experiência passível de estimativas e julgamentos que pode ser percebida de forma subjetiva ou objetiva. Subjetiva por variar de indivíduo para indivíduo de acordo com determinado julgamento ou interpretação e objetiva através do relato verbal de dor ou por meio de sinais indicativos de alteração fisiológica e comportamental (GUINSBURG R e CUENCA MC, 2010).

Dentre os parâmetros fisiológicos de dor, os mais utilizados para a avaliação de fenômenos dolorosos na prática clínica são a saturação de oxigênio, a pressão arterial sistólica e as frequências cardíaca e respiratória. Os parâmetros fisiológicos apesar de úteis, em geral, não podem ser usados de forma isolada na avaliação da dor no RN por se tratarem de parâmetros inespecíficos, que variam a depender da condição clínica do paciente (GUINSBURG R e CUENCA MC, 2010; OLIVEIRA AAS, 2016).

A avaliação comportamental trata-se de uma avaliação subjetiva da dor frente à resposta do RN a um estímulo doloroso. Dentre elas trabalha-se com a resposta motora à dor, a mímica facial, o choro, movimentos intensos de braço e pernas e o padrão de sono e vigília. Tais medidas se comparadas às alterações fisiológicas parecem representar uma resposta mais específica ao estímulo doloroso. Entretanto, por ser um 
parâmetro subjetivo, que depende da interpretação do observador, põe em risco sua precisão quanto a real mensuração do estímulo doloroso (GUINSBURG R e CUENCA MC, 2010; INSTITUTO FERNANDES FILGUEIRA, 2013).

Dentro desse contexto, faz-se necessário o conhecimento da dor para que estes parâmetros tanto fisiológicos quanto comportamentais sejam avaliados a fim de que, diante de situações dolorosas, seja possível atuar em prol da melhor condição de saúde para o RN. Essas escalas, portanto, têm importante função de realizar a "leitura" da resposta à dor do tipo comportamental, que são as escalas conhecidas como unidimensionais e as escalas multidimensionais, que incluem uma combinação de parâmetros objetivos e subjetivos relacionados à resposta à dor exibida pelo RN (GUINSBURG R e CUENCA MC, 2010)

Comparando as escalas unidimensionais com as multidimensionais, as primeiras tendem a ser mais sensíveis pelo fato de existir situações nas quais um RN pode apresentar indícios de dor do ponto de vista comportamental, mas não necessariamente apresentar alterações em seus parâmetros fisiológicos. Isso pode ser explicado por conta de a expressão de dor ocorrer predominantemente no núcleo do tronco cerebral, responsável pelos movimentos da face e corpo, e não nos controles centrais relativos às respostas fisiológicas. Por isso a importância de se utilizar a escala unidimensional comportamental como um dos vários instrumentos de avaliação de dor exibidos pelo RN (GUINSBURG R e CUENCA MC, 2010)

A escolha do tema do presente estudo surgiu, primeiramente, mediante a necessidade de tornar a assistência de saúde mais humanizada. Considerando os RN como uma população que é incapaz de comunicar-se verbalmente, faz-se imprescindível a adoção de instrumentos que avaliem a dor de forma satisfatória e que consiga atender as necessidades de saúde desta população.

Outra questão igualmente importante é a sensibilização da equipe acerca da temática dor. Atualmente sendo ela considerada como quinto sinal vital, a avaliação da mesma deve ser tão importante quanto outros parâmetros já bastante conhecidos da prática clínica, como temperatura, pressão arterial, pulso e respiração.

O objetivo deste estudo foi avaliar o conhecimento dos profissionais de saúde sobre o manejo e correta avaliação da dor em uma unidade neonatal.

\section{MÉTODOS}

Trata-se de um estudo descritivo de abordagem qualitativa realizado no período de maio a outubro de 2016, na UTIN em um Hospital Regional do Distrito Federal (DF). Esta pesquisa foi aprovada pelo comitê de ética sob número de parecer 1.621.521.

A pesquisa envolve a descrição de comportamentos ou aspectos a partir da análise individual da população estudada. Tal descrição exige determinado aprofundamento sobre a temática abordada, pois este é o fator crucial que a diferencia de outros tipos de estudos. Com a pesquisa qualitativa é possível obter maior aprofundamento da temática através da investigação de questões que necessitem ser melhor exploradas (RAUPP FM e BEUREN IM, 2003).

A unidade neonatal é composta pela UTIN, unidade de cuidado intermediário neonatal convencional (UCINCo) e a unidade de cuidado intermediário neonatal canguru (UCINCa) cada uma com 8, 10 e 6 leitos, respectivamente. Tal instituição além da UTIN possui a UTI adulto e pediátrica bem como bloco cirúrgico e centro obstétrico, serviços de ambulatório e emergência 24 horas.

A população do estudo foi constituída por profissionais da equipe de enfermagem que trabalham na UTIN de um hospital público do Distrito Federal. Foi aplicado termo de consentimento livre e esclarecido entre todas as participantes envolvidas na pesquisa.

Neste estudo foi realizado uma caracterização dos participantes da pesquisa, que foram identificados pela letra E, em algarismo arábico, conforme foram entrevistadas. Para Franchi CMGG (2012) as amostras de conveniência são comuns na área de saúde, sendo muito utilizada em unidades hospitalares e por muitas vezes a única maneira de estudar determinado problema. Tal amostra é formada por elementos que o pesquisador reuniu dada a disponibilidade do local do estudo. 
Foram excluídos os profissionais que estavam de licença médica, maternidade ou férias durante o período da pesquisa ou aqueles que não quiserem participar da pesquisa.

A coleta de dados foi composta por duas etapas. Na primeira etapa foi aplicado um questionário sobre dor a fim de identificar os conhecimentos prévios da equipe de enfermagem sobre o tema abordado. Posteriormente, foi realizado uma capacitação da equipe através de uma roda de conversa com a temática abordada.

No questionário foi possível obter características pessoais e profissionais, além de perguntas sobre o entendimento da dor de um modo geral e a dor neonatal em particular (Anexo 1). As respostas do questionário foram transcritas e organizadas. Para a análise desses dados foi aplicado o método de análise do conteúdo, utilizando a técnica de análise temática, em que o texto é dividido em unidades de significação. $A$ análise de conteúdo pelo método de Bardin L (2009) consiste em um conjunto de técnicas de investigação que, por meio de uma descrição objetiva, sistemática quantitativa e ou qualitativa do conteúdo manifesto das comunicações, tem por finalidade a interpretação destas. Divide-se em três etapas: a) pré-análise; b) exploração do material e c) tratamento dos resultados, inferência e interpretação.

Na segunda etapa da pesquisa foi realizado uma capacitação mediante uma roda de conversa com tópicos relacionados a dor propriamente dita, hospitalização do $\mathrm{RN}$, direitos do prematuro e a importância da aplicação da escala de dor existente na instituição.

\section{RESULTADOS}

Do quantitativo de 52 pessoas que compõe a equipe de enfermagem do local do estudo, 43 profissionais de enfermagem participaram do estudo, seguindo os critérios de inclusão da pesquisa. Todos profissionais abordados eram do sexo feminino, sendo a grande maioria delas técnicas de enfermagem $(83,8 \%)$, seguidas de enfermeiras $(11,6 \%)$ e residentes de enfermagem $(4,6 \%)$.

A porcentagem dos profissionais de enfermagem da pesquisa foi dividida em 5 faixas etárias. De um total de 43 entrevistas, houve predominância de profissionais entre 40 e 50 anos com 16 (37,3\%), seguida da faixa entre 30 a 40 anos alimentando a mesa porcentagem entre 50 a 60 anos com 10 (23,3\%), finalizando a porcentagem entre 20 a 30 anos com 7 (43\%).

Quanto ao tempo de atuação em UTIN, das 34 técnicas de enfermagem que responderam ao item, a média de trabalho corresponde aproximadamente 12,2 anos. Das enfermeiras foi equivalente a um tempo médio de 8 anos. E, por último, o trabalho das residentes de enfermagem corresponde a uma média de 1,7 anos em uma UTIN.

Das 43 profissionais de enfermagem que participaram do estudo, apenas 16,3\% responderam já terem feito algum tipo de capacitação em que o tema de dor em neonatologia foi abordado. Quanto a esta capacitação, 4 delas $(57,1 \%)$ alegaram o curso do Método Canguru como o responsável pela abordagem do assunto.

Quanto ao processo de análise de conteúdo resultou em 5 categorias, sendo elas: Categoria 1: Conhecimento sobre o conceito de dor; Categoria 2: Dor neonatal: é maior, menor ou igual ao adulto? Categoria 3: Dor como quinto sinal vital: como reconhecê-la?; Categoria 4: Medidas para alívio da dor; Categoria 5: Conhecimento sobre escalas de avaliação da dor, que serão discutidas a seguir.

\section{Categoria 1: conhecimento sobre o conceito de dor}

O primeiro questionamento do instrumento de coleta de dados foi sobre o que o entrevistado entendia por dor. Primeiramente, a dor foi por muitas vezes relacionada à sensação desagradável, como é possível observar nas seguintes respostas:

E13: "Dor é uma sensação desagradável que pode estar associada a trauma ou não". 
E22: "A dor é um desconforto físico que pode alterar os parâmetros vitais".

E33: "Sintoma bastante desconfortável caracterizado por sinais como taquicardia, hipertensão ou sinais flogísticos".

Houve também referências a dor como sinal vital nos depoimentos a seguir:

E4: "Dor pode ser considerada um sinal vital, pois interfere no organismo como um todo".

E15: "A dor é um sinal vital que indica alerta, quando o organismo não está em sua condição normal".

Durante o depoimento das entrevistadas também foi possível perceber que a dor por muitas vezes está ligada ao sofrimento e incômodo.

E34: "Incômodo, sofrimento, manifestação que algo está errado".

E25: "Condição incômoda sentida pelo indivíduo por condição patológica ou por intervenções realizadas pela equipe de saúde".

E8: "Algo que incomoda, que deveríamos aliviar o mais rápido possível".

Em algumas falas foi possível correlacionar a dor a estado de desequilíbrio do organismo frente a algum processo não fisiológico.

E29: "Dor é uma reação do organismo informando que algo está diferente do normal".

E30: "Se você sente dor em algum lugar é porque é um sinal de alerta para comunicar ou investigar o que está causando a dor".

E21: "Entendo que a dor é resultado de um organismo que não está em homeostasia. É um sintoma de que algo não está certo".

\section{Categoria 2: dor neonatal: é maior, menor ou igual ao adulto?}

Sobre o questionamento levantado se RN sentem menos dor que os adultos, $93 \%$ das profissionais de enfermagem alegaram que tão afirmação é falsa. Apenas duas afirmaram que acreditam que, de fato, eles sentem menos dor que o adulto. Uma delas justificou tal afirmativa com a seguinte resposta:

E12: " Acho que os receptores para dor são imaturos".

\section{Categoria 3: dor como quinto sinal vital: como reconhecê-la?}

O questionamento do instrumento de coleta de dados sobre a dor poder ser considerada um quinto sinal vital, apenas 7\% das entrevistadas responderam que não. Quantitativo este correspondente a categoria profissional de técnicas de enfermagem.

Quanto ao reconhecimento quando o RN está sentindo dor, o choro foi o sinal mais citados das entrevistas, seguido da expressão facial de dor, irritabilidade/inquietação e a taquicardia como uma alteração de sinal vital.

E13: "Choro persistente, expressão facial, irritabilidade, taquicardia".

E14: "Inquietação, choro excessivo, expressão facial de dor".

E21: "Choro forte e prolongado, expressão facial, aumento da frequência cardíaca".

\section{Categoria 4: medidas para alívio da dor}

Sobre medidas utilizadas no local do estudo para amenizar a dor no RN, a sucção não nutritiva associada a glicose $25 \%$ foi a mais citada, seguida do uso individual de glicose a $25 \%$ via oral, a contenção do RN como 
uma forma de promover sua organização comportamental foi a terceira medida mais citada, e, por último, a administração de medicação como os analgésicos e anestésicos, segundo prescrição médica.

\section{E32: "Uso de glicose e sucção não nutritiva que libera endorfina e diminui a dor".}

E27: "Medidas gerais de conforto: posicionamento, contenção, sucção não nutritiva ou com glicose a $25 \%$ ".

\section{E40: "Sedação (tanto venosa, via oral ou tópica)".}

\section{Categoria 5: conhecimento sobre escalas de avaliação da dor}

Nessa categoria, 79\% das participantes do estudo alegaram desconhecer escalas de avaliação em neonatal. $E$ das $21 \%$ que alegaram conhecer as mesmas, mais da metade destas referenciaram conhecer a escala facial de dor, que é mais utilizada na população adulta e pediátrica. Apenas duas participantes referenciaram a PIPP (Escala Perfil de Dor do Prematuro), a CRIES (Escore para a Avaliação da Dor PósOperatória do Recém-Nascido) e a NIPS (Escala de Dor Neonatal).

\section{DISCUSSÃO}

Nesta pesquisa toda a população de estudo foi composta por mulheres. Na enfermagem, de fato, o sexo feminino é predominante. Tal relação pode ser justificada pelo fato do cuidado estar historicamente atrelado a esta profissão (DONOSO MT, 2000).

Sobre a abordagem de algum tipo de capacitação em dor neonatal, o Método Canguru obteve destaque nas respostas das participantes. Tal método é uma proposta inovadora à assistência neonatal responsável por promover através da posição canguru inúmeros benefícios tanto para o RN quanto para seus genitores. Dentro das características do método destacam-se a estimulação para um contato pele a pele precoce, aproximação entre a mãe/pai e o bebê a fim de promover o vínculo afetivo e o estímulo à amamentação. Em relação ao $\mathrm{RN}$, o método favorece tanto a estabilidade de sua temperatura corporal, redução do estresse e da dor, diminuição do choro e das taxas de infecção hospitalar (BRASIL, 2013).

Capacitar é uma atitude importante para que os profissionais se sintam tecnicamente mais atualizados sobre determinada questão e sensibilizados para fornecer uma assistência em saúde mais qualificada e, acima de tudo, mais humana. No presente estudo, a capacitação permitiu a abordagem teórica de diversos temas relacionados ao universo do RN como direitos do prematuro - garantido por meio de declaração universal - e aspectos positivos e negativos de uma hospitalização precoce em RN's que nasceram antes da data prevista para o parto. Quanto a dor também foi possível discutir o que é a dor propriamente dita, medidas atenuantes para sua prevenção além de características comportamentais e fisiológicas demonstradas pelos RN's que dão indícios da presença de dor nessa população.

No contexto da dor neonatal, portanto, para garantir uma assistência humanizada e adequada aos RN, os profissionais responsáveis pelo cuidado aos mesmos devem ser treinados e capacitados de forma contínua para que a dor e, consequentemente, seu manejo, sejam sempre considerados (SOARES AC et al., 2016)

A Associação Internacional para o Estudo da Dor (IASP) definiu a dor como uma sensação negativa ligada ou não a algum dano tecidual provocado diretamente no corpo humano (IASP, 2010).

Comparando a dor entre RN's e adultos, por muito tempo acreditou-se que os primeiros eram incapazes de sentir dor com a mesma intensidade que os adultos por conta da mielinização incompleta do nervo nesta população.

Após o surgimento de novos estudos sobre o tema, percebeu-se que a diminuição das fibras mielinizadas se relaciona apenas com a velocidade na transmissão da dor e não com a dor em si. Esta inclusive pode ser percebida antes mesmo do nascimento do $\mathrm{RN}$ através do surgimento de terminações nervosas já na sétima semana de gestação e de conexões nervosas iniciadas ainda com doze semanas de gestação (TAMEZ RN, 2013). 
O reconhecimento da dor como $5^{\circ}$ sinal vital começou com James Campbell, Presidente da Sociedade Americana de Dor, em 1996. Seu principal objetivo foi conceder a mesma importância à dor assim como os outros sinais vitais (pressão arterial, temperatura, pulso e respiração). Tal atitude teria o objetivo de promover um tratamento mais adequado para a quem dela sofre seus efeitos e, consequentemente, elevar a conscientização por parte dos profissionais de saúde em se garantir um tratamento à dor apropriado sempre que necessário (SBED, 2017).

Para Bottega FH e Fontana RT (2010) medidas de intervenções para o alívio da dor estão relacionadas intrinsicamente com o cuidado prestado, visando sempre o atendimento de forma humanizada e atento às necessidades básicas da população. Medidas para avaliar e prevenir a dor, portanto, além de suprir uma demanda do indivíduo, têm a importante função de promover sua saúde diante de um ambiente hospitalar por muitas vezes hostil frente aos inúmeros procedimentos de saúde realizados.

Em um estudo realizado município de Fortaleza, Ceará, dentro dos parâmetros utilizados por profissionais da categoria de técnicas de enfermagem para avaliar a dor no $\mathrm{RN}$, os gestos faciais foram os mais citados, seguido do choro, irritação do RN, movimentos corporais, episódios de taquicardia e hipossaturação. Tais dados discordaram em poucos aspectos da presente pesquisa visto que à exceção da hipossaturação, todos os sinais de dor estiveram presentes (MENDES LC et al., 2013)

A utilização de práticas para o alívio da dor no RN é fundamental no cuidado prestado ao mesmo. Métodos farmacológicos e não farmacológicos são estratégias importantes no provimento de um cuidado mais humanizado e de qualidade. Tais métodos também são essenciais na prevenção de danos futuros à saúde dos RN como consequência da exposição à dor por muitas vezes de forma prolongada (MOTTA GC E CUNHA ML, 2015).

Intervenções não farmacológicas como soluções adocicadas, suç̧ão não nutritiva, amamentação, contato pele-pele (mãe e filho) e diminuição da estimulação tátil têm sido utilizadas para o alívio e manejo da dor durante procedimentos relacionados à dor aguda. São medidas eficazes, de baixo custo e risco para os RN (BRASIL, 2013).

Investigando o conhecimento das participantes sobre escalas de avaliação de dor neonatal, a maioria absoluta desconhecia tais instrumentos. Ao considerarmos que a maioria destas possuem mais de 10 anos trabalhando na mesma área, tal desconhecimento pode estar associado a uma falta de motivação segundo a Teoria dos Dois Fatores, do psicólogo Frederick Herzberg. Uma das categorias dessa teoria debate sobre os fatores intrínsecos que estimulam o trabalhador a desenvolver suas atividades e como ele se sente em relação ao cargo diante de aspectos como realização, reconhecimento, responsabilidade e progresso (RIBEIRO AV, 2011).

A educação permanente baseada na participação dos profissionais deve ser o principal caminho para que os fatores motivacionais sejam valorizados e melhores resultados sejam alcançados no âmbito do trabalho tanto individual quanto coletivo (GIORDANI AT et al., 2014). Faz-se necessário a realização de educação permanente no local do estudo para que os profissionais desenvolvam mais o conhecimento teórico acerca do tema.

Para o diagnóstico de dor não existe um instrumento ideal visto que todas as escalas são baseadas em métodos multidimensionais (comportamento, fisiologia e contexto do $\mathrm{RN}$ ). Através da aplicação de tais instrumentos a dor pode ser quantificada e, consequentemente, condutas possam ser tomadas para um tratamento mais correto e direcionado.

Previamente a aplicação de qualquer instrumento, faz-se importante o treinamento dos profissionais de saúde responsáveis por esta função como também a sensibilização dos mesmos visto a natureza subjetiva da dor (BUENO M, 2013).

Tal estudo apesar da quantidade reduzida de profissionais, o que caracteriza uma limitação, a semelhança entre os discursos obtidos facilitou o entendimento do leitor em identificar os conhecimentos dos profissionais de enfermagem na avaliação da dor neonatal em uma unidade de terapia intensiva. 


\section{CONCLUSÃO}

De acordo com os resultados encontrados na presente pesquisa quanto a identificação dos conhecimentos prévios das profissionais de enfermagem sobre a dor neonatal, pôde-se constatar que o tema é pouco debatido entre os profissionais frente aos baixos índices de capacitação realizados sobre a temática, fato que também justifica a falta de conhecimento, por parte da maioria dos profissionais, sobre as escalas de avaliação de dor específicas na área de neonatal. Sobre o entendimento de dor pôde-se perceber, de fato, o seu caráter subjetivo, mas que por muitas vezes o conceito de dor, de uma maneira geral, apareceu de forma semelhante nos depoimentos obtido das participantes. Tal semelhança permitiu inclusive uma junção em palavras-chave a fim de facilitar essa percepção pelo leitor. Sobre a dor ser considerada quinto sinal vital, reconhecimento da dor no RN e se caso presente, medidas corretivas para amenizá-la, a pesquisa obteve resultados satisfatórios diante das respostas dadas pelas participantes do estudo. Este fato é muito pertinente, pois percebe-se que apesar do pouco debate teórico sobre o tema, na prática, as profissionais de enfermagem mostraram-se preparadas em identificar a dor e em intervir com medidas de prevenção para o seu alívio. Portanto, a aplicação do questionário sobre dor permitiu identificar, previamente, o conhecimento da equipe de enfermagem sobre $o$ assunto e assim trabalhar conforme as reais necessidades demonstradas. Através da capacitação também foi possível sensibilizar a equipe dando-lhes ferramentas para identificar a dor corretamente no $\mathrm{RN}$ e realizar medidas para seu alívio o mais precocemente possível.

\section{REFERÊNCIAS}

1. BARDIN L. Análise de conteúdo. Lisboa: Edições 70; 2009, 200p.

2. BRASIL. Conselho Nacional de Saúde. Resolução nํ 466, de 12 de dezembro de 2012. Disponível em: http://conselho.saude.gov.br/resolucoes/2012/reso466.pdf. Acesso em: 19 de janeiro de 2015.

3. BRASIL. Ministério da Saúde. Secretaria de Atenção à Saúde. Departamento de Ações Programáticas Estratégicas. Atenção à Saúde do recém-nascido: guia para os profissionais de saúde. Brasília: Ministério da Saúde, 2014. Disponível em: http://bvsms.saude.gov.br/bvs/publicacoes/atencao saude recem nascido v1.pdf. Acesso em: 19 de Março de 2015.

4. BRASIL. Ministério da Saúde. Secretaria de Atenção à Saúde. Departamento de Ações Programáticas Estratégicas. Atenção humanizada ao recém-nascido de baixo peso: Método Canguru/ Ministério da Saúde, Secretaria de Atenção à Saúde, Departamento de Ações Programáticas Estratégicas. - 2. ed. - Brasília: Editora do Ministério da Saúde, 2013. Disponível em: http://bvsms.saude.gov.br/bvs/publicacoes/atencao humanizada recem nascido canguru.pdf. Acesso em: $16 \mathrm{de}$ Fevereiro de 2015.

5. BOTTEGA FH, FONTANA RT. A dor como quinto sinal vital: utilização da escala de avaliação por enfermeiros de um hospital geral. Texto Contexto Enfermagem 2010;19(2):283-90.

6. BUENO M. Métodos simples para avaliar a dor. Enfermagem Revista: Dor Neonatal. São Paulo, 2013. Disponível em: http://www.coren-sp.gov.br/sites/default/files/13-dor neonatal.pdf. Acesso em 10 de janeiro de 2017.

7. DIAS FS, MARBA ST. Avaliação da dor prolongada no recém-nascido: adaptação da escala EDIN para a cultura brasileira. Texto Contexto Enfermagem. 2014;23(4):964-70

8. DONOSO MT. O gênero e suas possíveis repercussões na gerência de enfermagem. Revista Mineira Enfermagem 2000;4(1/2):67-9.

9. FORTUNATO JG, et al. Escalas de dor no paciente crítico: uma revisão integrativa. Revista HUPE, Rio de Janeiro. 2013;12(3):110-7.

10. GIORDANI AT, et al. Adesão da equipe de enfermagem à higienização das mãos: fatores motivacionais. Rev Rene. 2014;15(4):559-68.

11. GUINSBURG R, CUENCA MC. A linguagem da dor no recém-nascido. Documento Científico do Departamento de Neonatologia. Sociedade Brasileira de Pediatria. São Paulo, 2010.

12. IASP. Associação Internacional para o Estudo da Dor. Guia para o Tratamento da Dor em Contextos de Poucos Recursos. Seattle; 2010.

13. INSTITUTO FERNANDES FILGUEIRA. Atenção à Saúde do Recém-nascido: Superando pontos críticos. Módulo 1: Dor. Disciplina de Telemedicina do Departamento de Patologia da Faculdade de Medicina da USP. São Paulo, 2013. Disponível em: http://www.iff.fiocruz.br/pdf/1 dor2014.pdf. Acesso em 9 de janeiro de 2017.

14. FRANCHI CMGG. Apostila de Bioestatística. Curso Tecnologia em Radiologia Médica. Bioestatística. Brasil; 2012. 
15. MENDES LC, et al. A dor no recém-nascido na unidade de terapia intensiva neonatal. Revista Enfermagem UFPE. 2013;7(11):6446-54.

16. MOTTA GC, CUNHA ML. Prevenção e manejo não farmacológico da dor no recém-nascido. Revista Brasileira Enfermagem 2015;68(1):131-5.

17. OLIVEIRA AAS. Avaliação da dor em população especial: população pediátrica e neonatal. Centro de Tratamento Intensivo Neonatal do Instituto da Criança. Programa de educação continuada em fisiopatologia e terapêutica da dor. São Paulo, 2016.

18. RAUPP FM, BEUREN IM. Como elaborar trabalhos monográficos em contabilidade: teoria e prática. Metodologia da pesquisa aplicável às ciências sociais. São Paulo: Atlas, p. 76-97, 2003. Disponível em: http://www.geocities.ws/cienciascontabeisfecea/estagio/Cap_3_Como_Elaborar.pdf. Acesso em 9 de janeiro de 2017.

19. RIBEIRO AV. Teorias motivacionais. Pontifícia Universidade Católica de Minas Gerais. Campus de Poços de Caldas. Minais Gerais, 2011.

20. SANTOS LM et al. Identificação e tratamento da dor no recém-nascido prematuro na Unidade de Terapia Intensiva. Revista Brasileira Enfermagem, 2012; 65(2): 269-75.

21. SBED. Sociedade Brasileira para Estudo da Dor. Capítulo Brasileiro da Internacional Association for Study of Pain IASP. 5 Sinal Vital. São Paulo, 2016. Disponível em: http://www.sbed.org.br/materias.php?cd_secao=65. Acesso em 20 de dezembro de 2016.

22. SOARES AC, et al. Dor em unidade neonatal: conhecimento, atitude e prática da equipe de enfermagem. Cogitare Enfermagem. 2016;21(2):1-10.

23. TAMEZ RN. Enfermagem na UTI Neonatal: Assistência ao recém-nascido de Alto Risco. 5a ed. Rio de Janeiro, Guanabara Koogan, 2013, 110p. 